\title{
Comparing low-pass sequencing and genotyping for trait mapping in pharmacogenetics
}

\author{
Kaja Wasik', Tomaz Berisa', Joseph K. Pickrell', Jeremiah H. Li ${ }^{1 *}$ (D, Dana J. Fraser ${ }^{2}$, Karen King ${ }^{2}$ and Charles Cox ${ }^{3}$
}

\begin{abstract}
Background: Low pass sequencing has been proposed as a cost-effective alternative to genotyping arrays to identify genetic variants that influence multifactorial traits in humans. For common diseases this typically has required both large sample sizes and comprehensive variant discovery. Genotyping arrays are also routinely used to perform pharmacogenetic (PGX) experiments where sample sizes are likely to be significantly smaller, but clinically relevant effect sizes likely to be larger.

Results: To assess how low pass sequencing would compare to array based genotyping for PGx we compared a low-pass assay (in which 1x coverage or less of a target genome is sequenced) along with software for genotype imputation to standard approaches. We sequenced 79 individuals to $1 \times$ genome coverage and genotyped the same samples on the Affymetrix Axiom Biobank Precision Medicine Research Array (PMRA). We then down-sampled the sequencing data to $0.8 x, 0.6 x$, and $0.4 x$ coverage, and performed imputation. Both the genotype data and the sequencing data were further used to impute human leukocyte antigen (HLA) genotypes for all samples. We compared the sequencing data and the genotyping array data in terms of four metrics: overall concordance, concordance at single nucleotide polymorphisms in pharmacogenetics-related genes, concordance in imputed HLA genotypes, and imputation $\mathrm{r}^{2}$. Overall concordance between the two assays ranged from $98.2 \%$ (for $0.4 \mathrm{x}$ coverage sequencing) to $99.2 \%$ (for $1 \times$ coverage sequencing), with qualitatively similar numbers for the subsets of variants most important in pharmacogenetics. At common single nucleotide polymorphisms (SNPs), the mean imputation $r^{2}$ from the genotyping array was 0.90 , which was comparable to the imputation $r^{2}$ from $0.4 x$ coverage sequencing, while the mean imputation $r^{2}$ from $1 \times$ sequencing data was 0.96 .
\end{abstract}

Conclusions: These results indicate that low-pass sequencing to a depth above $0.4 x$ coverage attains higher power for association studies when compared to the PMRA and should be considered as a competitive alternative to genotyping arrays for trait mapping in pharmacogenetics.

Keywords: Trait mapping, Low-pass sequencing, Pharmacogenetics, Genotype imputation

* Correspondence: jeremy@gencove.com

${ }^{1}$ Gencove, Inc., New York, NY 10016, USA

Full list of author information is available at the end of the article

(c) The Author(s). 2021 Open Access This article is licensed under a Creative Commons Attribution 4.0 International License, which permits use, sharing, adaptation, distribution and reproduction in any medium or format, as long as you give appropriate credit to the original author(s) and the source, provide a link to the Creative Commons licence, and indicate if changes were made. The images or other third party material in this article are included in the article's Creative Commons licence, unless indicated otherwise in a credit line to the material. If material is not included in the article's Creative Commons licence and your intended use is not permitted by statutory regulation or exceeds the permitted use, you will need to obtain permission directly from the copyright holder. To view a copy of this licence, visit http://creativecommons.org/licenses/by/4.0/ The Creative Commons Public Domain Dedication waiver (http://creativecommons.org/publicdomain/zero/1.0/) applies to the data made available in this article, unless otherwise stated in a credit line to the data. 


\section{Background}

Research in human genetics relies on efficiently profiling the genome of large numbers of individuals. A number of approaches can be used for this, usually trading off comprehensiveness (i.e. the fraction of the genome that is measured) with cost. By far the most commonly-used approach is the genotyping array, in which a set of known polymorphisms (usually around 500,000-2,000,000) is measured. This technology is inexpensive (currently on the order of tens to hundreds of dollars), but the set of genetic variants profiled is a small number of all known variants, and the technology does not allow for the detection of new (for example rare or population-specific) genetic variants. Genotyping arrays are commonly used for pharmacogenetics (PGx) studies where typically sample numbers are more limited, but inclusion of PGx focused variants on the arrays makes them suitable tools for screening the genome for markers associated with efficacy and adverse events $[3,15,16]$.

The technological alternative to genotyping technology is sequencing technology, in which specific polymorphisms are not targeted for analysis, but rather the entire genome is sampled with some average depth of coverage. As sequencing costs have dropped, low-pass sequencing (for our purposes, which we will define as sequencing in which the average coverage of the genome is equal to or lower than $1 \mathrm{x}$ ) becomes an appealing alternative to genotyping $[4,6,14]$. As an intuition for why this approach is useful, note that a human sample sequenced at $0.4 \mathrm{x}$ coverage is expected to have a single sequencing read covering each of around 28 million of the 84.7 million genetic variants identified in the 1000 Genomes Project [1], while a genotyping array obtains measurements (albeit somewhat less noisy measurements) at two orders of magnitude fewer sites.

In this paper, we directly compare genotyping results from low-pass sequencing to a commonly used genotyping array, the Affymetrix Axiom Biobank Precision Medicine Research Array (PMRA). Two types of metrics are relevant for this comparison. One is simply the genome-wide coverage of the assay, which we measure using average imputation quality. The other is genotyping quality at particular genetic variants of interest. We were particularly interested in applications to PGx-the identification of genetic variants that influence drug response. In this application, genetic variants in the major histocompatibility complex (MHC) and genes involved in drug metabolism (so-called "ADME" genes, for absorption, distribution, metabolism, and excretion) are known to be particularly relevant. We thus considered these separately.

\section{Results}

We selected 79 individuals to be both genotyped and sequenced. These individuals derive from a pool of volunteers based out of Cambridge, UK for which prior consent was obtained. Each individual was genotyped on the Affymetrix Axiom Biobank PMRA, and sequenced by Gencove, Inc. to an average of $1 x$ coverage using the Illumina HiSeq 4000 platform with paired-end 150 base pair reads. Sequencing reads were then sampled at random to obtain an average of $0.8 \mathrm{x}, 0.6 \mathrm{x}$, and $0.4 \mathrm{x}$ coverage of the genome (Methods).

We then performed genotyped imputation of genetic variants in the 1000 Genomes Phase 3 release. This imputation was performed using minimac2 (for the genotyping array data) or Gencove's loimpute software v0.18 (for the low-pass sequencing data, see Methods for details). Both the unimputed PMRA data and the imputed low-pass sequencing data were then used to impute HLA genotypes using HIBAG [18].

The relevant metrics to use when comparing the two technologies depend on the downstream use cases. Specifically, if an investigator is interested in identifying genetic variants associated with a trait but has no a priori knowledge of where in the genome such variants are likely to be located, then the relevant metric is the average correlation between imputed genotype calls and true genotypes. On the other hand, if the investigator knows that specific variants are most likely to be relevant to the trait of interest, then the relevant metric is the concordance between the technologies at those specific sites. Since in PGx applications there are some specific genes and variants of interest, we computed metrics in both of these classes.

\section{Overall genotype concordance}

We first examined the overall concordance between the genotyping arrays and imputed sequences at different depths. To do this, we removed genotypes imputed with low confidence (with less than $90 \%$ posterior probability on a single genotype), and assessed the concordance between the two platforms, averaging across individuals, using metrics from the draft guidance of the United States Food and Drug Administration [19]. These metrics measure concordance for variants present and absent in a reference genome-a "positive percent agreement" (PPA) for variants that are different from the reference and a "negative percent agreement" (NPA) for variants that match a reference genome. For our purposes we considered the genotypes from the PMRA as "truth"; in this case the PPA ranged from $98.2 \%$ for $0.4 x$ coverage sequencing to $99.2 \%$ for $1 \mathrm{x}$ coverage sequencing, while the NPA ranged from $99.8 \%$ for $0.4 x$ coverage to $99.9 \%$ for $1 \mathrm{x}$ coverage (Table 1 ).

\section{Genotype concordance at ADME genes}

We then specifically compared the concordance between the genotypes at variants in ADME genes as defined by 
Table 1 Genotype concordance between genotyping and sequencing platforms

\begin{tabular}{llll}
\hline Comparison & PPA (\%) & NPA (\%) & No Calls (Average) \\
\hline Accuracy, .4x vs PMRA & $98.22 \%$ & $99.82 \%$ & 2535 \\
Accuracy, .6x vs PMRA & $98.76 \%$ & $99.85 \%$ & 1848 \\
Accuracy, .8x vs PMRA & $99.01 \%$ & $99.86 \%$ & 1508 \\
Accuracy, 1x vs PMRA & $99.19 \%$ & $99.88 \%$ & 1251 \\
\hline
\end{tabular}

In all cases the genotyping array was treated as 'Truth'. Positive \% Agreement (PPA)- The percent of non-reference calls in the Truth dataset detected by Test, ignoring no calls in Test. (True Positives / True Positives + False Negatives). Negative \% Agreement (NPA) - The percent of reference calls in the Truth dataset detected by Test, ignoring no calls in Test. (True Negatives / True Negatives + False Positives). No Calls- Count of No Calls in test that were variant in Truth. No calls are averaged across all 79 individuals. The total number of overlapping variants between the PMRA and the imputed sequence data is $\sim 423 \mathrm{k}$

Hoverlson et al. [7]. There were 216 such variants that were directly genotyped on the PMRA. We thus computed the same concordance metrics specifically at these 216 variants. For these analyses we excluded lowconfidence genotype calls from the low-pass sequencing data; the percentage of excluded calls range from 1.6\% of genotype calls in the $0.4 \mathrm{x}$ data down to $0.8 \%$ of genotype calls in the $1 \mathrm{x}$ data.

Concordance results are presented in Fig. 1a. At common variants (where the minor allele is present in more than five copies in the sample, corresponding to a minor allele frequency over 3\%), PPA ranged from 98.5\% (for $0.4 \mathrm{x}$ coverage) up to $99.4 \%$ (for $1 \mathrm{x}$ coverage). The lowest concordance metric was the PPA at rarer variants, which ranged from $82.1 \%$ (for $0.4 \mathrm{x}$ coverage) to $95.2 \%$ (for $1 \mathrm{x}$ coverage).

\section{Genotype concordance at HLA}

Apart from ADME genes, another important locus in PGx is the MHC region. We imputed four digit HLA alleles from both the PMRA and sequencing data using HIBAG [18], and assessed the concordance across the two platforms at each of the seven HLA genes assessed by HIBAG. (Fig. 1b). There was little variation in imputed genotype concordance across levels of sequencing coverage, and with the exception of the gene DPB1, concordance was above $95 \%$.

For samples where we saw consistent discordance for a given gene between the platforms, we then generated gold standard HLA genotype calls (Methods). A total of 15 HLA genotype calls in 12 samples were retested in this manner. The correct calls were obtained at $7 / 15$ genotypes from the PMRA, and 6, 7, 7, and 8/16 genotypes after imputation from $0.4 \mathrm{x}, 0.6 \mathrm{x}, 0.8 \mathrm{x}$, and $1 \mathrm{x}$ sequencing, respectively.

\section{Imputation quality and comparison}

Finally, an important metric of how well a technology assays known polymorphisms in the genome is the

\section{A. Concordance at variants in ADME genes}

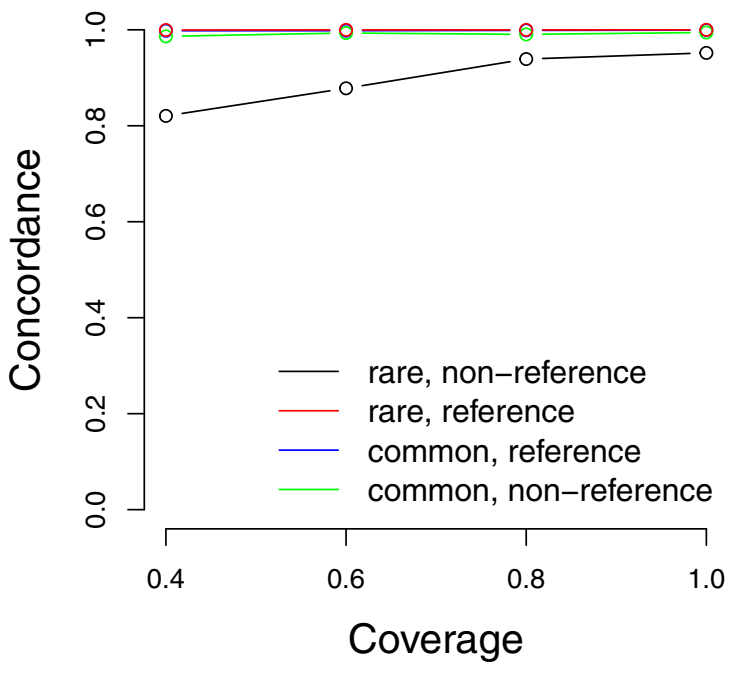

B. Concordance in HLA genotype calls

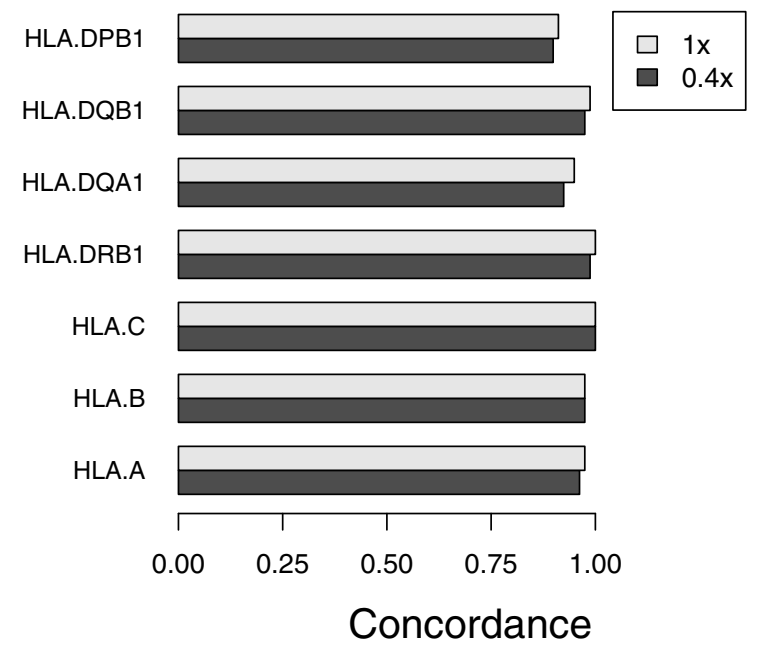

Fig. 1 Genotype concordance across platforms at specific variants relevant to pharmacogenomics. a. Concordance at SNPs in ADME genes. Variants were classified as "rare" if the minor allele was present in five or fewer copies in the sample (corresponding to an allele frequency of about 3\%. Concordance rates are split according to the genotype calls on the PRMA, which was considered "truth" - reference concordance is at variants where the PRMA is homozygous reference, and non-reference concordance is for all other sites. b. Concordance in HLA genotypes across platforms. Shown are the concordance rates between sequencing and genotyping array data in imputed HLA genotypes. Concordance is shown for $0.4 x$ and $1 x$ sequencing

squared correlation between imputed genotype dosages and the true genotypes (known as "imputation $\mathrm{r}^{2}$ "). Intuitively, if the researcher has a flat prior on where in the genome to look for an association between a genetic variant and a trait, the average squared correlation is a measure of the power of the study. 
We computed this metric for different levels of sequencing coverage by correlating the imputed allelic dosages with directly genotyped sites. We computed this same metric for the genotype data by using the leaveone-out $r^{2}$ at genotyped sites computed by minimac 2 . At common variants (allele frequency $>5 \%$ in the cohort), the average $r^{2}$ obtained from the genotyping array was 0.9 (Fig. 2), consistent with previous reports from a European population [13]. For the sequencing data, this metric varied from 0.91 (for $0.4 x$ coverage) to 0.96 (for $1 \mathrm{x}$ coverage). When all variants across the frequency spectrum were considered, the difference in average $\mathrm{r}^{2}$ between assays was even more pronounced, with the average $\mathrm{r}^{2}$ for the genotyping array being 0.85 and the average $r^{2}$ for the sequencing data ranging from 0.88 (for $0.4 x$ coverage) to 0.93 (for $1 x$ coverage).

To investigate the effect of the choice of imputation reference panel on imputation performance on the sequencing data, we performed a head-to-head comparison between using the 1000 Genomes and a subset of the Haplotype Reference Consortium (HRC) haplotypes [12] as reference panels using the above methodology (i.e., by treating the array data as "truth" and comparing overlapping sites between the reference panel and the array sites). Using the HRC dataset as the imputation reference panel yielded marginal increases in average $r^{2}$ values in all minor allele frequency (MAF) bins but the lowest, where it suffered a decrease of about 0.036 as

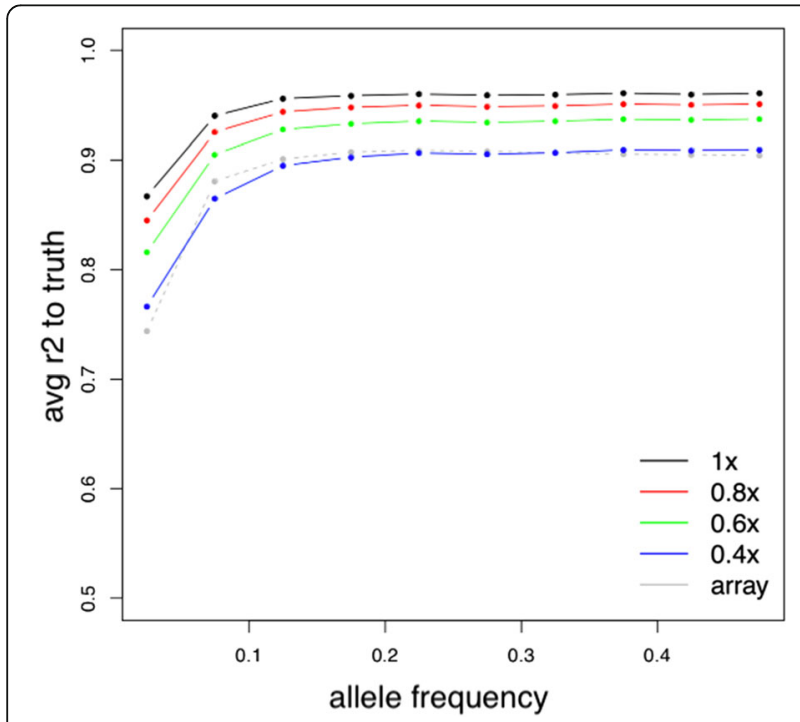

Fig. 2 Comparison of imputation quality across platforms. Alleles were binned according to their minor allele frequency (as measured on the genotyping array) and imputation $r^{2}$ averaged across all variants in the bin. For sequencing data, the array data was treated as 'truth' and imputation $r^{2}$ computed by correlating imputed dosages to array genotypes. For the array data, imputation $r^{2}$ for all genotyped variants was computed using a leave-one-out procedure implemented in minimac2 compared to the 1000 Genomes imputed sites in the same bin (Supp. Tables S1, S2). The exact details and further discussion on this particular comparison can be found in the accompanying Supplementary Materials (Supp. Figs. S1, S2).

\section{Discussion}

In this paper, we performed a direct comparison between low-pass sequencing (combined with imputation) and a commonly-used genotyping array for the purposes of trait mapping in pharmacogenetics.

We observed that overall, genotype calls across the two platforms were highly concordant, with a positive percent agreement (PPA) of the imputed sequence data to the genotyping array calls ranging from $98.22 \%$ at $0.4 x$ to $99.19 \%$ at $1 x$ coverage.

At ADME genes, we observed qualitatively similar results, with a PPA ranging from 98.5 to $99.4 \%$ at sites of common variation ( $>3 \%$ minor allele frequency in this cohort) and a PPA ranging from 82.1 to $95.2 \%$ at rarer variants $(<3 \%)$.

Four-digit HLA alleles in the MHC region imputed from sequencing data had high concordance with those imputed from the PMRA, with all concordances across the range of sequencing coverage observed to be above 95\% with the exception of those in the DPB1 gene; further validation using a gold-standard assay of the HLA genotype calls resulted in similar concordance results between the imputed sequence or PMRA data and the resulting gold standards.

For the purposes of trait mapping, low-pass sequencing above a sequencing coverage of $0.4 \mathrm{x}$ had higher overall imputation accuracy as measured by imputation $\mathrm{r}^{2}$ than the genotyping array, indicating a corresponding increase in power.

Beyond simply comparing concordance between assays, it is important to consider other, orthogonal considerations when deciding between low-pass sequencing and genotyping arrays for PGx purposes. For instance, due to the sheer number of measurements (reads) made during a sequencing run (even at very low coverages), sequence data allows far more sensitive detection of copy number and structural variation $[5,20]$. As intuition, consider that sequencing a human genome at a depth of $0.1 \mathrm{x}$ using $150 \mathrm{bp}$ reads yields 2.2 million reads corresponding to measurements at $330 \mathrm{Mbp}$, compared to a typical genotyping array which generates point measurements at only a few hundreds of thousands to a couple million sites.

Similarly, sequencing affords the capability to perform metagenomic profiling and analysis of the microbiome via analysis of non-human sequencing reads deriving from a DNA sample, as well as analysis of mitochondrial count $[2,17]$. 
Logistical and budgetary considerations are also essential in real-world project planning. In a number of scenarios with different outcomes and study designs, lowpass sequencing has been shown to increase performance, for example by increasing the effective sample size of a genome-wide association study [14] or increasing the accuracy of polygenic risk scoring [10].

One of the drawbacks of low-pass sequencing compared to genotyping arrays is that while the average performance over the entire genome is consistently higher with sequencing, there may occasionally be a subset of specific SNPs or genes that, for a given study design, must be assayed with a level of accuracy and precision which low-coverage sequencing is simply unable to provide. In order to address this, it may be useful to develop an assay which combines lowpass whole genome sequencing with higher-depth coverage of pre-selected target regions on the genome, such that a single sequencing run simultaneously yields low-coverage sequence data across the genome at the same time as assaying specific SNPs, variants, or genes of interest with clinical grade accuracy.

\section{Conclusion}

As research into the effects of genetics on drug response continues to accelerate, it will become increasingly important for assays used in pharmacogenetics to provide reliable measurements both across the entire genome and at specific PGx focused variants. Our results demonstrate that low-pass sequencing and imputation provide a competitive alternative to genotyping arrays in both of these applications.

It is worth noting that the cost of sequencing is declining rapidly; if sequencing a human genome to $30 x$ coverage costs $\$ 1000$, then the cost of sequencing a human sample to $0.4 \mathrm{x}$ coverage is around $\$ 13$. The key components of cost in a low-pass sequencing assay then become sequencing library preparation and analysis. As the costs of sequencing continue to drop, the importance of these latter costs will continue to grow.

\section{Methods}

\section{Genotyping}

Samples were from study TMT109167: A study to collect blood samples from members of the Clinical Unit Cambridge, UK (CUC) volunteer panel for DNA extraction and storage, for investigation of prospective genotype-phenotype relationships and stratification of subjects for recruitment into future clinical trials.

DNA samples were genotyped by BioStorage Technologies/Bioprocessing Solutions Alliance, Brooks
Life Sciences (Piscataway, NJ, USA) using the Affymetrix Axiom PMRA.

Prior to genotype imputation, variants in each GWAS dataset were excluded using standard Affymetrix QC thresholds for the PMRA, if there were deviations from Hardy-Weinberg proportions within subgroups of any given ancestry or showed gross and irreconcilable differences in alleles or allele frequency with reference panel genotypes from the HapMap or 1000 Genome projects. Standard Affymetrix array QC sample level thresholds were also applied prior to imputation.

(http://www.affymetrix.com/support/downloads/ manuals/axiom_genotyping_solution_analysis_guide.pdf)

\section{Imputation of PMRA data}

Genotype imputation for genetic variants that were not directly genotyped ("untyped variants") was performed using a cosmopolitan haplotype reference panel from the 1000 Genomes Project [The 1000 Genomes Project Consortium, 2015], and using Hidden Markov Model methods as implemented in $\mathrm{MaCH}$ and minimac $[8,9]$.

\section{HLA genotyping}

High resolution HLA genotyping was performed at BioStorage Technologies/Bioprocessing Solutions Alliance, Brooks Life Sciences (Piscataway, NJ, USA) using the Thermo Fisher AllSet+ Gold SSP High-Resolution HLA kit for HLA-A, HLA-B, HLA-DRB1, HLA-DQB1 and HLA-DPB1 following the manufacturer's instructions.

\section{Sequencing}

Sequencing libraries were prepared from DNA using the KAPA Library Preparation Kit by Roche and sequenced on an lllumina HiSeq 4000 instrument. Sequencing reads for each sample were aligned to the genome using bwa mem [9], and sequencing reads were randomly sampled to obtain different levels of sequencing coverage. Imputation of genotypes from sequencing data was done using loimpute v. 0.18 by Gencove, Inc. (New York, NY) to a reference panel comprising a subset of the 1000 Genomes Phase 3 (described in more detail in the Supplementary Materials).

\section{Imputation of sequencing data}

Imputation was performed using an implementation of the Li and Stephens model [11], described in more detail in the Supplementary Note.

\section{Abbreviations}

PGx: Pharmacogenetics; PMRA: Precision Medicine Research Array;

HLA: Human leukocyte antigen; ADME: Absorption, distribution, metabolism, and excretion; SNP: Single nucleotide polymorphism; MHC: Major

histocompatibility complex; PPA: Positive percent agreement; NPA: Negative percent agreement; MAF: Minor allele frequency; HRC: Haplotype Reference Consortium 


\section{Supplementary Information}

The online version contains supplementary material available at https:/doi. org/10.1186/s12864-021-07508-2.

Additional file 1: Supplementary note. Details of the model underlying loimpute, the software used to impute the low-pass sequencing data analysed in this study.

Additional file 2: Supplementary materials. Description of imputation performance using the HRC as a haplotype reference panel rather than the 1000 Genomes Phase 3 release. Figure S1. Comparison of imputation $r^{2}$ across allele frequency bins for the 1000 Genomes panel. Figure S2. Comparison of imputation $r^{2}$ across allele frequency bins for the HRC panel.

\section{Acknowledgements}

The abstract from an early version of this study was presented at the $51 \mathrm{st}$ European Society of Human Genetics Conference (see https://www.nature. com/articles/s41431-019-0407-4\#Sec270)

\section{Authors' contributions}

KW, TB, JKP, and CC designed the study. KW, DJF, and KK performed the experiments. TB, JKP, JHL, DJF, KK, and CC analyzed the data. All authors reviewed the manuscript. The authors read and approved the final manuscript.

\section{Funding}

Not applicable.

\section{Availability of data and materials}

The datasets generated and/or analysed during the current study are proprietary and are therefore not publicly available. The data were not consented for use outside the scope of the present study, and as such, dataset access cannot be applied for.

Details on the 1000 Genomes Phase 3 release dataset and instructions on how to download the data can be found at the following URL: https://www. internationalgenome.org/category/phase-3/

Details on the Haplotype Reference Consortium datasets and instructions on how to apply for and access the data can be found at the following URL: http://www.haplotype-reference-consortium.org/data-access

\section{Declarations}

\section{Ethics approval and consent to participate}

Ethical approval (LREC ref.: 08/H0302/100, UK) and written informed consent were obtained prior to conducting this study. Namely, ethics approval was granted by National Research Ethic Service, Cambridgeshire 2 Research Ethics Committee, Victoria House, Capital Park, Fulbourn, Cambridge. CB21 5XB on 2nd Dec 2008. They are part of The Research Ethics Service (RES) affiliated with the Health Research Authority (HRA), based in the UK.

\section{Consent for publication}

Not applicable.

\section{Competing interests}

K. W., T.B., J.K.P., and J.H.L were employees of Gencove, Inc. at the time of writing.

\section{Author details}

'Gencove, Inc., New York, NY 10016, USA. 2PAREXEL Genomic Medicine, Durham, NC 27713, USA. ${ }^{3}$ GlaxoSmithKline, Stevenage, UK.

Received: 6 May 2020 Accepted: 5 March 2021

Published online: 20 March 2021

\section{References}

1. Auton A, Abecasis GR, Altshuler DM, Durbin RM, Abecasis GR, Bentley DR, Chakravarti A, Clark AG, Donnelly P, Eichler EE, et al. A global reference for human genetic variation. Nature. 2015;526(7571):68-74. https://doi.org/10.1 038/nature15393.
2. Cai N, Li Y, Chang S, Liang J, Lin C, Zhang X, Liang L, Hu J, Chan W, Kendler KS, Malinauskas T, Huang GJ, Li Q, Mott R, Flint J, et al. Genetic control over mtDNA and its relationship to major depressive disorder. Curr Biol. 2015;25(24):3170-7. https://doi.org/10.1016/j.cub.2 015.10.065.

3. Caldwell MD, Awad T, Johnson JA, Gage BF, Falkowski M, Gardina P, Hubbard J, Turpaz Y, Langaee TY, Eby C, King CR, Brower A, Schmelzer JR, Glurich I, Vidaillet HJ, Yale SH, Qi Zhang K, Berg RL, Burmester JK, et al. CYP4F2 genetic variant alters required warfarin dose. Blood. 2008;111(8): 4106-12. https://doi.org/10.1182/blood-2007-11-122010.

4. CONVERGE consortium, Cai N, Bigdeli TB, Kretzschmar W, Li Y, Liang J, Song L, Hu J, Li Q, Jin W, et al. Sparse whole-genome sequencing identifies two loci for major depressive disorder. Nature. 2015;523:588-91.

5. Dong Z, Zhang J, Hu P, Chen H, Xu J, Tian Q, Meng L, Ye Y, Wang J, Zhang M, Li Y, Wang H, Yu S, Chen F, Xie J, Jiang H, Wang W, Choy $\mathrm{KW}, \mathrm{Xu} Z$ Z. Low-pass whole-genome sequencing in clinical cytogenetics: a validated approach. Genet Med. 2016;18(9):940-8. https://doi.org/10.1038/gim.2015.199 Epub 2016 Jan 28. Erratum in: Genet Med. 2017 Jan;19(1):129. PMID: 26820068.

6. Gilly, A., Kuchenbaecker, K., Southam, L., Suveges, D., Moore, R., Melloni, G., Hatzikotoulas, K., Farmaki, A.-E., Ritchie, G., Schwartzentruber, J., et al. (2017). Very low depth whole genome sequencing in complex trait association studies.

7. Hovelson DH, Xue Z, Zawistowski M, Ehm MG, Harris EC, Stocker SL, Gross AS, Jang I-J, leiri I, Lee J-E, et al. Characterization of ADME gene variation in 21 populations by exome sequencing: Pharmacogenet. Genomics. 2017;27:89-100.

8. Howie B, Fuchsberger C, Stephens M, Marchini J, Abecasis GR. Fast and accurate genotype imputation in genome-wide association studies through pre-phasing. Nat Genet. 2012;44(8):955-9. https://doi.org/10.1038/ng.2354.

9. Li H, Durbin R. Fast and accurate short read alignment with burrowswheeler transform. Bioinformatics. 2009;25(14):1754-60. https://doi.org/10.1 093/bioinformatics/btp324.

10. Li JH, Mazur CA, Berisa T, Pickrell JK. Low-pass sequencing increases the power of GWAS and decreases measurement error of polygenic risk scores compared to genotyping arrays. Genome Res. 2021. gr-266486.

11. Li N, Stephens M. Modeling linkage disequilibrium and identifying recombination hotspots using single-nucleotide polymorphism data. Genetics. 2003;165(4):2213-33.

12. McCarthy S, Das S, Kretzschmar W, Delaneau O, Wood AR, Teumer A, Min Kang H, Fuchsberger C, et al. A reference panel of 64,976 haplotypes for genotype imputation. Nat Genet. 2016:48(10):1279-83. https://doi.org/10.1038/ng.3643.

13. Nelson, S.C., Romm, J.M., Doheny, K.F., Pugh, E.W., and Laurie, C.C. (2017). Imputation-based genomic coverage assessments of current genotyping arrays: Illumina HumanCore, OmniExpress, multi-ethnic global array and sub-arrays, global screening array, Omni2.5M, Omni5M, and Affymetrix UK biobank.

14. Pasaniuc B, Rohland N, McLaren PJ, Garimella K, Zaitlen N, Li H, Gupta N, Neale BM, Daly MJ, Sklar P, Sullivan PF, Bergen S, Moran JL, Hultman CM, Lichtenstein P, Magnusson P, Purcell SM, Haas DW, Liang L, Sunyaev S, Patterson N, de Bakker PIW, Reich D, Price AL, et al, Extremely low-coverage sequencing and imputation increases power for genome-wide association studies. Nat Genet. 2012;44(6):631-5. https:// doi.org/10.1038/ng.2283.

15. SEARCH Collaborative Group, Link E, Parish S, Armitage J, Bowman L, Heath S, Matsuda F, Gut I, Lathrop M, Collins R. SLCO1B1 variants and statininduced myopathy--a genomewide study. N Engl J Med 2008;359(8):789799. doi: https://doi.org/10.1056/NEJMoa0801936. Epub 2008 Jul 23. PMID: 18650507

16. Shuldiner AR, O'Connell JR, Bliden KP, et al. Association of cytochrome P450 2C19 genotype with the antiplatelet effect and clinical efficacy of Clopidogrel therapy. JAMA. 2009;302(8):849-57. https://doi.org/10.1001/ja ma.2009.1232

17. Wood DE, Lu J, Langmead B. Improved metagenomic analysis with kraken 2. Genome Biol. 2019). https://doi.org/10.1186/s13059-019-1891-0;20(1):257.

18. Zheng X, Shen J, Cox C, Wakefield JC, Ehm MG, Nelson MR, Weir BS. HIBAG—HLA genotype imputation with attribute bagging. Pharma J. 2014; 14(2):192-200. https://doi.org/10.1038/tpj.2013.18.

19. U.S. Food \& Drug Administration. (2018) Considerations for Design, Development, and Analytical Validation of Next Generation Sequencing (NGS) - Based In Vitro Diagnostics (IVDs) Intended to Aid in the Diagnosis 
of Suspected Germline Diseases. Retrieved from https://www.fda.gov/ media/99208/download

20. Zhou B, Ho SS, Zhang X, Pattni R, Haraksingh RR, Urban AE. Whole-genome sequencing analysis of CNV using low-coverage and paired-end strategies is efficient and outperforms array-based CNV analysis. J Med Genet 2018;

55(11):735-743. doi: https://doi.org/10.1136/jmedgenet-2018-105272. Epub 2018 Jul 30. PMID: 30061371.

\section{Publisher's Note}

Springer Nature remains neutral with regard to jurisdictional claims in published maps and institutional affiliations.

Ready to submit your research? Choose BMC and benefit from:

- fast, convenient online submission

- thorough peer review by experienced researchers in your field

- rapid publication on acceptance

- support for research data, including large and complex data types

- gold Open Access which fosters wider collaboration and increased citations

- maximum visibility for your research: over $100 \mathrm{M}$ website views per year

At $\mathrm{BMC}$, research is always in progress.

Learn more biomedcentral.com/submissions 